in vivo 35 : $993-998(2021)$

doi:10.21873/invivo.12342

\title{
Weak-evidence Fusion Candidates Detected by a FusionPlex Assay Using the Ion Torrent System
}

\author{
SHIRO FUJTA ${ }^{1,2}$, KATSUHIRO MASAGO ${ }^{1}$, EIICHI SASAKI ${ }^{1}$, SATOSHI TSUKUSHI ${ }^{3}$, \\ YOSHITSUGU HORIO ${ }^{4}$, HIROAKI KURODA ${ }^{5}$ and TOYOAKI HIDA ${ }^{4}$ \\ ${ }^{I}$ Department of Pathology and Molecular Diagnostics, Aichi Cancer Center, Nagoya, Japan; \\ ${ }^{2}$ Department of Respiratory Medicine, Kobe Central Hospital, Kobe, Japan; \\ ${ }^{3}$ Department of Orthopaedic Surgery, Aichi Cancer Center, Nagoya, Japan; \\ ${ }^{4}$ Department of Thoracic Oncology, Aichi Cancer Center, Nagoya, Japan; \\ ${ }^{5}$ Department of Thoracic Surgery, Aichi Cancer Center, Nagoya, Japan
}

\begin{abstract}
Background/Aim: The Archer FusionPlex platform is widely used for comprehensive fusion-gene detection in cancer tissues. This platform separately displays results for strong-evidence and weak-evidence fusion candidates (WEFCS). Distinctive fusion patterns are frequently found in the weak-evidence category and information about the patterns is clinically essential. Patients and Methods: We describe the type and frequency of WEFCs observed using the FusionPlex Sarcoma Panel ( $S$ Panel) and the FusionPlex ALK, RET, and ROS1 ver2 Panel (ARR Panel). Results: A total of 97 specimens were examined and 620 candidates were detected and categorized as WEFCs. A median of five WEFCs were detected per sample. In the S Panel group, there were 13 WEFCs with a frequency of more than 1\%. In the ARR Panel group, a total of 16 WEFCs were detected with a frequency of more than 1\%. Conclusion: Specific WEFCs were detected according to the panel selected.
\end{abstract}

Fusion gene formation is one of the major gene events that can drive malignancies in humans (1). Since imatinib was first approved for BCR-ABL-positive chronic myeloid leukemia (2), specific inhibitors of fusion gene function have become one of the mainstays of treatment for malignancies in humans. In addition, the detection of fusion genes can not

This article is freely accessible online.

Correspondence to: Shiro Fujita, MD, Ph.D., Department of Respiratory Medicine, Kobe Central Hospital, 2-1-1 Soyama-cho, Kita-ku, Kobe, Hyogo, 651-1145, Japan. Tel: +81 785942211, e-mail: jp.shirofujita@gmail.com

Key Words: Next-generation sequencing, non-small cell lung cancer, sarcoma, fusion gene, Ion Torrent, FusionPlex. only lead to the precise diagnosis of a tumor but also predict prognosis and treatment responses $(1,3)$.

Classically, fusion gene searches have been performed using fluorescence in situ hybridization (FISH) and/or quantitative real-time polymerase chain reaction (RT-PCR), however, these modalities are often insufficient. Unlike firstline targeted therapy, subsequent therapies are ideally determined on the basis of information about the presence or location of a secondary mutation in the kinase domain of the fusion gene, which requires sequencing of the kinase domain. In other words, it is not only the presence of the fusion gene but also the determination of the fusion partner that is necessary, otherwise it is impossible to design suitable primers to analyze secondary mutations. In clinical practice it is often the case that only formalin-fixed paraffinembedded (FFPE) samples are available to search for fusion genes. However, varying degrees of RNA degradation and denaturation in FFPE specimens are unavoidable.

One solution to the above limitations is the use of the rapid amplification of cDNA ends (RACE) method. As the presence of a fusion gene is predicted from tumor histology and clinical information, primers are placed on the expected characteristic sequence of the fusion gene, and cDNA is synthesized from the 5' side. In recent years, techniques have been developed to apply RACE to massively parallel sequencing. A number of manufacturers have proposed a method of designing primers based on the characteristic sequence information of the expected fusion genes, synthesizing cDNA using this primer set, and determining the sequence of the fusion gene using next-generation sequencing (NGS).

In the FusionPlex platform (ArcherDx, Inc., Boulder, CO, USA), anchored multiplex PCR chemistry utilizes the RACE method to identify gene fusions (4). Primers are set on known 3' and 5' sites and cDNA is synthesized from both sides, to detect rare fusion partners. FASTQ sequence data is then obtained by NGS on the Illumina ${ }^{\circledR}$ or Ion Torrent ${ }^{\mathrm{TM}}$ 


\begin{tabular}{|c|c|}
\hline Step & Main task \\
\hline RNA extraction and quantification & Required amount of RNA: $1500 \mathrm{ng} / \mathrm{chip}$ \\
\hline Library preparation & Following the manufacturer's instructions. \\
\hline Chip loading & $\begin{array}{l}\text { Semiautomated chip loading system; up to } \\
\text { two chips can be prepared. }\end{array}$ \\
\hline Sequencing & Ion Torrent S5 sequencer. \\
\hline $\begin{array}{l}\text { Fusion detection using Archer } \\
\text { Analysis software }\end{array}$ & $\begin{array}{l}\text { Automated data analysis. The analytical } \\
\text { workflow is shown in Figure } 2 .\end{array}$ \\
\hline
\end{tabular}

Figure 1. Workflow for gene fusion analysis of clinical samples using the FusionPlex platform.

platforms, and subsequent analysis is conducted in the Archer Analysis Pipeline, which is available as either cloudbased or semi-automated software that can be installed on a stand-alone computer. The uploaded FASTQ (Illumina) or BAM (Ion Torrent) file is processed and automatically analyzed by the software.

Annotation of each fusion consensus is performed by aligning the obtained sequencing to the human genome using BLAST. The quality of these alignments depends on the alignment length and repeat-content of the sequence. The resulting annotation are output in two categories: Strongevidence fusion candidates and weak-evidence fusion candidates. The manufacturer recommends referring to strong evidence candidates. However, when deciding on a treatment method in clinical settings, if there is no fusion equivalent to strong-evidence fusion candidates, we are forced to refer to weak-evidence fusion candidates.

In our experience, a distinctive fusion pattern is frequently found in this weak-evidence category. Here, we describe information about weak-evidence fusion candidates when performing sequence analysis using the Ion Torrent system. This information is considered to be particularly useful for practitioners performing analyses using the FusionPlex platform.

\section{Patients and Methods}

Ethics. This study was approved by the Aichi Cancer Center Hospital's Institutional Review Board. We obtained written informed consent from all participants. The study was conducted in accordance with the ethical principles of the Declaration of Helsinki.
Patient information. Tumor samples were collected from Aichi Cancer Center Hospital, Japan, between March 2018 and November 2019. Since July 2017, we have conducted assays using the FusionPlex platform. In non-small cell lung cancer (NSCLC) samples, EGFR and KRAS gene mutations were searched using the cycleave PCR method, and the ALK status was examined by immunohistochemistry (IHC). For samples that were negative using all these tests, we searched for other fusion genes where interventions may be possible, such as ROS1 and NTRK1, using the FusionPlex ALK, RET, and ROS1 ver2 Panel (ARR Panel). In sarcoma cases where a histologically definitive diagnosis could not be made by hematoxylin and eosin staining, immunostaining, and FISH examination, fusion genes were searched for using the FusionPlex Sarcoma Panel (S Panel). From March 2018, a method using diluted sodium hydroxide was recommended for library preparation in FusionPlex, and the efficiency of library preparation was improved. This study was limited to assays from this time.

If the same fusion gene detected as a weak-evidence fusion gene repeatedly appears in different samples, the candidate fusion gene is likely to be a false positive. However, we cannot exclude the possibility that the detected weak-evidence fusion genes actually exist in the sample. Therefore, we separately analyzed samples that were highly suspected of giving false positive results; only the samples in which there was strong evidence for a fusion gene and the result was confirmed by another modality (FISH and RT-PCR) were identified in our database, and the fusion gene information with weak evidence in these samples was collected separately.

IHC and FISH. Tissue sections (4- $\mu$ m thick) were cut from FFPE blocks and placed on a charged slide. The sections were deparaffinized in xylene and rehydrated using a graded series of ethanol solutions. They were then subjected to immunohistochemical staining with a mouse monoclonal primary anti-ALK antibody (clone 5A4, ready to use) using the Dako EnVision ${ }^{\mathrm{TM}}$ FLEX detection system (Dako/Agilent Technologies, Santa Clara, CA, USA). 


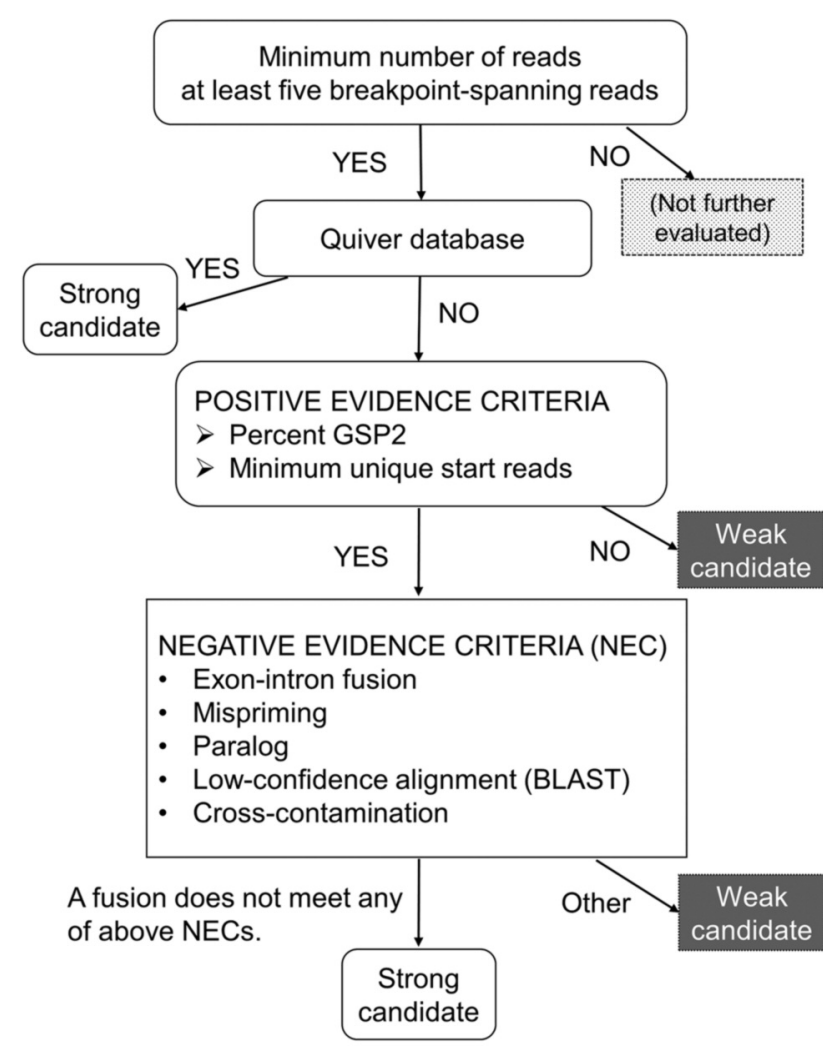

Figure 2. A flow chart showing the processing performed by the Archer Analysis software. Details of positive evidence criteria and negative evidence criteria are described in Table I and Table II, respectively.

FISH with a dual-color, break-apart probe was performed. ALK, EWSR1, RET, ROS1, and SS18 FISH (Vysis ALK BreakApart FISH Probe Kit, Visys LSI EWSR1 Break-Apart FISH Probe Kit, Vysis RET Break-Apart FISH Probe Kit, Vysis ROS1 Break-Apart FISH Probe Kit, and Vysis LSI SS 18 Break Apart FISH Probe Kit; Abbott Molecular, Chicago, IL, USA) were performed to confirm the results obtained by the FusionPlex assay. To test for TEF3 gene rearrangement, a TFE3 break-apart probe (SureFISH TFE3 3' RD FISH Probe and SureFISH TFE3 5' GR FISH Probe, Agilent Technologies) was used. For each probe, 100 randomly selected nonoverlapping tumor cell nuclei were examined. When separate red and green signals were observed, a split signal for the probe was considered positive. The cutoff values were set to $>10 \%$.

Purification of DNA and RNA from FF and FFPE tissues. RNA and DNA were derived from either FFPE sections or fresh-frozen samples. Total RNA was extracted from FFPE sections using the Maxwell RSC RNA FFPE Kit and Maxwell RSC Instrument (Promega, Madison, WI, USA). RNA extraction from fresh-frozen samples was performed using the RNeasy Mini Kit (Qiagen, Hilden, Germany), as per the manufacturer's instructions. DNA was purified from FFPE samples using a QIAamp DNA FFPE Tissue Kit (Qiagen). DNA purification from the fresh-frozen samples was performed using a QIAamp DNA Mini Kit (Qiagen).
Table I. Positive selection criteria: a fusion must meet all the following criteria to be a strong-evidence fusion candidate.

Positive selection criteria

Percent gene specific primer 2 (GSP2): Percent GSP2 is the proportion of breakpoint-spanning reads that support the candidate relative to the total number of RNA reads spanning the breakpoint. Percent GSP2 needs to be at least $10 \%$ for a fusion to be considered a strong-evidence fusion candidate.

Minimum unique start sites: Within the population of breakpoint-spanning reads that support the candidate, there will be a distribution of unique start sites. There must be at least three unique start sites for a fusion to be considered a strong-evidence fusion candidate.

The cycleave PCR and RT-PCR methods. The methodological details of the cycleave PCR and RT-PCR methods are described elsewhere $(5,6)$. We used a chimeric DNA-RNA-DNA probe labeled with a fluorescent dye and a quencher at either end. An RNA sequence of the probes corresponding to those of the wild type and point mutations (EGFR Exon 21 L858R and KRAS Exon 2 G12X) were labeled with FAM and ROX, respectively. When mutant molecules are present in a sample and PCR-amplified DNA generates a complete hybrid with the RNA portion of the mutant probe, RNase-H cuts the probe at the RNA-DNA heteroduplex into two pieces, leading to a significant increase in fluorescence intensity by separating the fluorescent dye from the quencher. Common fragment analysis was used to detect the deletion in exon 19 of the EGFR gene. Sample DNA was amplified with a FAM-labeled primer set and PCR products were electrophoresed. PCR was used to amplify the shorter segment of DNA, creating a new peak in the electropherogram if a deletion mutation was present.

RT-PCR with sequencing was performed for fusion gene alterations (except for $A L K, E W S R 1, R E T, R O S 1, S S 18$, and TEF3), as previously described (7-9). In brief, total RNA was reverse transcribed to cDNA and then subjected to amplification with fusion-specific primers. Gel electrophoresis was performed with follow-up Sanger sequencing on PCR products with visible bands.

The FusionPlex platform. The system is based on a target enrichment approach, using the RACE method (Figure 1). The methodological details are described elsewhere $(4,10)$. In brief, a minimum of $200 \mathrm{ng}$ of total RNA was converted to cDNA. This cDNA was subjected to end repair, adenylation, and ligation with a universal adaptor. Following these procedures, the cDNA was amplified by two rounds of nested PCR using gene-specific primers (GSP1 and GSP2) and a universal adaptor. During these steps, the cDNA was purified several times using Agencourt ${ }^{\circledR}$ AMPure $^{\circledR}$ XP beads (Beckman Coulter, Inc., Brea, CA, USA). Libraries were quantified using the Applied Biosystems StepOne Real-Time PCR system and pooled to equimolar concentration. Emulsion PCR and chip loading were performed using the Ion Chef system, according to the manufacturer's recommendations (Thermo Fisher Scientific, Inc., Waltham, MA, USA). Sequencing was performed using semiconductor chips on an Ion Torrent S5 (Thermo Fisher Scientific). In this study, we used semiconductor chips from an Ion 540 Chip Kit.

The raw data were processed and automatically analyzed using Archer Analysis software version 6.0 (ArcherDx, Inc.), at default parameters. Human Genome build GRCh37 (hg19) was used. The 
Table II. Negative selection criteria: A fusion must not meet any of the following criteria to be called a strong-evidence fusion candidate.

\section{Negative selection criteria}

Exon-intron fusion: If the fusion sequence on one side of the breakpoint is found to be entirely intronic (which is indicative of a DNA mispriming event), the fusion will be classified as exon-intronic.

Mispriming: If significant sequence similarity is found between fusion partners, the event is likely to be due to mispriming. Additionally, if the fusion breakpoint is less than $3 \mathrm{bp}$ from the GSP2, then similarity is assumed.

Paralogs: Archer Analysis compares the identities of the fusion partners with a list of known paralogs taken from the Ensembl database.

Low confidence: Annotation of each fusion consensus is performed by aligning the sequencing to the human genome with BLAST. The quality of these alignments, and the confidence of the resulting annotation, depends on the alignment length and the repeat content of the sequence. Cross-contamination: If a low-expressing fusion candidate shows significant similarity to a high-expressing fusion candidate in the same analysis, it will be considered the result of intra-run cross-contamination.

Table III. Patients' clinical information.

\begin{tabular}{lc}
\hline Characteristics & Number \\
\hline Age (mean) & 13 to 85 (57.8) \\
$\quad$ Unknown cases & 11 \\
Gender & 47 \\
$\quad$ Male & 42 \\
Female & 8 \\
$\quad$ Unknown cases & \\
Types of specimen & 74 \\
FFPE & 23 \\
Fresh frozen tissue & \\
Histology & 52 \\
Sarcoma & 45 \\
Non-small cell lung cancer & \\
Purpose of assay & 87 \\
Diagnostic purpose & 10 \\
Validation assay & \\
Specimen obtained & 83 \\
Operation & 13 \\
Biopsy & 1 \\
Cell block & \\
\hline
\end{tabular}

determination of fusion gene candidates was performed according to the flowchart shown in Figure 2. For any candidate to be considered, there had to be at least five breakpoint-spanning reads that supported the candidate. If there were less than five, it was not evaluated any further. Next, it was determined whether a fusion gene existed in the Quiver database of known fusions (http://quiver.archerdx.com). If a fusion was found in Quiver, it was considered to be a strong candidate. If a fusion was not found in Quiver, then the fusion was examined based on positive evidence criteria (Table I) and negative evidence criteria (Table II), and classified as either a strong-evidence candidate or a weak-evidence candidate.
Table IV. Total number and detection frequency of weak-evidence fusion candidates in all cases analyzed using the S Panel.

\begin{tabular}{lcc}
\hline Fusion gene & Number* & Percent ${ }^{\dagger}$ \\
\hline FOXO1-FOXO3 & 121 & 35.9 \\
FOXO1-LINC00598 & 36 & 10.7 \\
FUS-EWSR1 & 19 & 5.6 \\
TAF15-FUS & 18 & 5.3 \\
CIC-SH3RF3 & 9 & 2.7 \\
FUS-PDE3A & 9 & 2.7 \\
INTS4-STAT6 & 9 & 2.7 \\
ZNF254-STAT6 & 8 & 2.4 \\
EWSR1-PHACTR4 & 5 & 1.5 \\
FUS-TAF15 & 5 & 1.5 \\
TFG-ZNF254 & 5 & 1.5 \\
UTRN-STAT6 & 5 & 1.5 \\
YWHAE-FUS & 4 & 1.2 \\
\hline
\end{tabular}

*Number of weak-evidence fusion genes detected (total 336); †The percentage of 336 weak-evidence candidate genes (S panel) is shown.

Table V. Total number and detection frequency of weak-evidence fusion candidates in all cases analyzed using the ARR Panel.

\begin{tabular}{lcc}
\hline Fusion gene & Number* & Percent $^{\dagger}$ \\
\hline LTK-ALK & 62 & 21.8 \\
DIP2C-ALK & 31 & 10.9 \\
FZR1-ALK & 23 & 8.1 \\
LRP1-ALK & 20 & 7.0 \\
ZNF254-RET & 12 & 4.2 \\
INTS4-ROS1 & 10 & 3.5 \\
LDLRAD2-RET & 9 & 3.2 \\
PID1-ALK & 5 & 1.8 \\
SFTPB-ALK & 5 & 1.8 \\
ANKRD30BL-ROS1 & 4 & 1.4 \\
CDC42BPG-ALK & 4 & 1.4 \\
DYNC2H1-ROS1 & 4 & 1.4 \\
PCBD2-ROS1 & 4 & 1.4 \\
SIRT3-ALK & 4 & 1.4 \\
MROH7-ALK & 3 & 1.1 \\
ZNF337-RET & 3 & 1.1 \\
\hline
\end{tabular}

*Number of weak-evidence fusion genes detected (total 284); †The percentage of 284 weak-evidence candidate genes (ARR panel) is shown.

\section{Results}

From March 2018 to November 2019, 97 specimens were tested with the FusionPlex assay. Clinical characteristics of the evaluated cases are shown in Table III. Of these specimens, 52 were sarcoma samples, examined using the $\mathrm{S}$ Panel. The remaining 45 specimens were examined by the ARR Panel in cases where NSCLC was negative for $E G F R$ and $K R A S$ gene mutations and also negative for $A L K$ gene rearrangement. Eleven specimens were from external 
Fujita et al: Weak-evidence Fusion Candidates Using FusionPlex

Table VI. Total number and detection frequency of weak-evidence fusion candidates in the $S$ Panel cases where the driver fusion gene was determined using another method (FISH or RT-PCR).

\begin{tabular}{lrr}
\hline Fusion gene & Number* $^{*}$ & Percent $^{\dagger}$ \\
\hline FOXO1-FOXO3 & 58 & 33.3 \\
FOXO1-LINC00598 & 21 & 12.1 \\
FUS-EWSR1 & 9 & 5.2 \\
INTS4-STAT6 & 7 & 4.0 \\
TAF15-FUS & 7 & 4.0 \\
ZNF254-STAT6 & 7 & 4.0 \\
CIC-SH3RF3 & 3 & 1.7 \\
DLG2-STAT6 & 3 & 1.7 \\
FUS-PDE3A & 3 & 1.7 \\
THTPA-TFG & 3 & 1.7 \\
UTRN-STAT6 & 3 & 1.7 \\
FUS-HFM1 & 2 & 1.1 \\
FUS-PAPSS1 & 2 & 1.1 \\
FUS-TAF15 & 2 & 1.1 \\
TFG-ZNF254 & 2 & 1.1 \\
YWHAE-FUS & 2 & 1.1 \\
\hline
\end{tabular}

*Number of weak-evidence fusion genes detected (total 174); the percentage of 174 weak-evidence candidate genes (S panel) is shown.

institutions and there was insufficient information about the clinical characteristics of these patients (e.g., age and/or gender).

With regard to observed fusion genes categorized as weakevidence fusion candidates, a total of 620 candidates (159 types) were detected. A median of 5 (range: 0 to 37) weakevidence fusion candidates was detected per sample. In the S Panel group, there were 13 weak-evidence candidates with a frequency of more than $1 \%$ (Table IV). In the ARR Panel group, 16 weak-evidence candidates were detected with a frequency of more than $1 \%$ (Table $\mathrm{V}$ ).

There were 34 samples in which strong-evidence fusion candidate were found, and in which the presence of the fusion gene was confirmed with another modality, such as FISH. For these 34 samples, the fusion gene had already been separately confirmed as a driver gene alteration, and it was highly likely that the fusion genes detected with weak evidence levels were false positives. In the S Panel group, driver fusion genes were confirmed in 29 samples and there were 174 weak-evidence fusion candidates. Table VI shows weak-evidence candidates with a frequency of more than $1 \%$. The weak-evidence fusion candidates listed in Table IV are also listed in Table VI, supporting the notion that the weak-evidence fusion candidates found in Table IV were highly likely to be false positives. With regard to the ARR Panel group, driver fusion genes were confirmed in five samples and there were 31 weak-evidence fusion candidates. Observed weak-evidence fusion candidates are shown in Table VII.
Table VII. Total number and detection frequency of Weak Evidence fusion candidates in the ARR Panel cases where the driver fusion gene was determined using another method (FISH or RT-PCR).

\begin{tabular}{lcc}
\hline Fusion gene & Number* $^{*}$ & Percent $^{\dagger}$ \\
\hline LTK-ALK & 7 & 22.6 \\
DIP2C-ALK & 4 & 12.9 \\
FZR1-ALK & 4 & 12.9 \\
LRP1-ALK & 4 & 12.9 \\
ZNF254-RET & 3 & 9.7 \\
INTS4-ROS1 & 1 & 3.2 \\
LDLRAD2-RET & 1 & 3.2 \\
LOC101927625-ALK & 1 & 3.2 \\
PCBD2-ROS1 & 1 & 3.2 \\
PID1-ALK & 1 & 3.2 \\
SFTPB-ALK & 1 & 3.2 \\
UNC45B-ROS1 & 1 & 3.2 \\
USP53-ROS1 & 1 & 3.2 \\
\hline
\end{tabular}

*Number of weak-evidence fusion genes detected (total 31); †The percentage of 31 weak-evidence candidate genes (ARR panel) is shown.

\section{Discussion}

This close examination of FusionPlex assay results revealed many fusion candidates, some of which were seen repeatedly. Furthermore, the profiles of weak-evidence fusion candidates in the samples with proven driver-gene rearrangements were consistent with those of weak-evidence candidates in the whole group. In the FusionPlex system, all FASTQ data output from a sequencer were analyzed by dedicated software. In fact, the analytical pipeline relied entirely on Archer. The parameters of the software can be adjusted by the user, but the supplier recommends using the default settings. In addition, the obtained read has unique tag sequences, and this tag information cannot be disclosed because it is confidential. Therefore, the FASTQ files cannot be handled as they are as RNAseq data. For these reasons, it is necessary to carefully confirm the analysis results. When using the FusionPlex system to analyze an actual sample, weak-evidence fusion candidates that we have previously presented need to be carefully interpreted, especially if they are repeatedly observed. We recommend that these weakevidence candidates be validated with RT-PCR or another modality before including the results in clinical reports.

In this study, completely different weak-evidence fusion candidates were detected by the ARR and S Panels, which suggests that the weak candidates detected are substantially different depending on the panel selected for the assay. In addition, the supplier currently offers products that can select a probe for a fusion gene search with a complete custom specification. When using such a custom panel, detected weak-evidence fusion candidates are considered to be 
different, depending on the selected probes. Therefore, when performing an assay using a new panel, it is advisable to perform several test-runs to obtain information about weakevidence fusion candidates.

Illumina has the highest share of the high-throughput sequencing market; however, we used the Ion Torrent sequencer instead of the Illumina sequencer. These two sequencers have different assay principles, and the FASTQ files obtained as pair-end and single-end are also different, so the weak-evidence fusion candidates also differ. In addition, the software provided by Archer for analyzing raw data is also being upgraded, so the displayed analysis results will change as the analysis software improves.

In conclusion, when the fusion gene assay was performed on the FusionPlex Platform using the Ion Torrent sequencer, it was revealed that specific weak-evidence fusion candidates were displayed according to the panel selected. For researchers using the platform, it is recommended to perform several test-runs in advance, on samples for which fusion gene information is already known, to obtain information about weak-evidence fusion candidates.

\section{Conflicts of Interest}

No competing financial interests exist in relation to this study.

\section{Authors' Contributions}

SF and KM designed the study. SF, KM and ES performed all experiments. SF, KM and ST analyzed the results. YH, HK and TH contributed to the interpretation of the results. SF wrote the article with contributions from KM, ES, ST and YH. All Authors have read and approved the final article.

\section{Acknowledgements}

This work was supported by the Japan Society for the Promotion of Science (JSPS) KAKENHI (grants JP15H10017 to SF).

\section{References}

1 Mitelman F, Johansson B and Mertens F: The impact of translocations and gene fusions on cancer causation. Nat Rev Cancer 7: 233-245, 2007. PMID: 17361217. DOI: 10.1038/nrc2091

2 Druker BJ: Imatinib as a paradigm of targeted therapies. Adv Cancer Res 91: 1-30, 2004. DOI: 10.1016/S0065-230X(04) 91001-9
3 Kumar-Sinha C, Kalyana-Sundaram S and Chinnaiyan AM: Landscape of gene fusions in epithelial cancers: Seq and ye shall find. Genome Med 7, 2015. DOI: 10.1186/s13073-015-0252-1

4 Zheng Z, Liebers M, Zhelyazkova B, Cao Y, Panditi D, Lynch KD, Chen J, Robinson HE, Shim HS, Chmielecki J, Pao W, Engelman JA, Iafrate AJ and Le LP: Anchored multiplex PCR for targeted next-generation sequencing. Nat Med 20: 14791484, 2014. PMID: 25384085. DOI: 10.1038/nm.3729

5 Yatabe $\mathrm{Y}$, Hida $\mathrm{T}$, Horio $\mathrm{Y}$, Kosaka T, Takahashi $\mathrm{T}$ and Mitsudomi T: A rapid, sensitive assay to detect EGFR mutation in small biopsy specimens from lung cancer. J Mol Diagnostics 8: 335-341, 2006. PMID: 16825506. DOI: $10.2353 /$ jmoldx. 2006.050104

6 Fujita S, Masago K, Takeshita J, Okuda C, Otsuka K, Hata A, Kaji R, Katakami N and Hirata Y: Validation of an ion torrent sequencing platform for the detection of gene mutations in biopsy specimens from patients with non-small-cell lung cancer. PLoS One 10: e0130219, 2015. PMID: 26076009. DOI: 10.1371/journal.pone.0130219

7 Takeuchi K, Soda M, Togashi Y, Suzuki R, Sakata S, Hatano S, Asaka R, Hamanaka W, Ninomiya H, Uehara H, Lim Choi Y, Satoh Y, Okumura S, Nakagawa K, Mano H and Ishikawa Y: RET, ROS1 and ALK fusions in lung cancer. Nat Med 18: 378 381, 2012. PMID: 22327623. DOI: 10.1038/nm.2658

8 Boyle TA, Masago K, Ellison KE, Yatabe Y and Hirsch FR: ROS1 immunohistochemistry among major genotypes of nonsmall-cell lung cancer. Clin Lung Cancer 16: 106-111, 2015. PMID: 25467930. DOI: 10.1016/j.cllc.2014.10.003

9 Takeuchi K, Choi YL, Soda M, Inamura K, Togashi Y, Hatano S, Enomoto M, Takada S, Yamashita Y, Satoh Y, Okumura S, Nakagawa K, Ishikawa Y and Mano H: Multiplex reverse transcription-PCR screening for EML4-ALK fusion transcripts. Clin Cancer Res 14: 6618-6624, 2008. PMID: 18927303. DOI: 10.1158/1078-0432.CCR-08-1018

10 Lam SW, Cleton-Jansen AM, Cleven AHG, Ruano D, van Wezel T, Szuhai K and Bovée JVMG: Molecular analysis of gene fusions in bone and soft tissue tumors by anchored multiplex PCR-based targeted next-generation sequencing. J Mol Diagnostics 20: 653-663, 2018. PMID: 30139549. DOI: 10.1016/j.jmoldx.2018.05.007

Received December 12, 2020

Revised December 28, 2020

Accepted December 29, 2020 